Z Gerontol Geriat 2012 · 45:692-693

DOI 10.1007/s00391-012-0405-4

Online publiziert: 25 . November 2012

(c) Springer-Verlag Berlin Heidelberg 2012

\author{
M. Marschollek ${ }^{1} \cdot$ C. Becker ${ }^{2}$ \\ ${ }^{1}$ Peter-L.-Reichertz-Institut für Medizinische Informatik der Technischen Universität Braunschweig \\ und der Medizinischen Hochschule Hannover \\ ${ }^{2}$ Klinik für Geriatrische Rehabilitation, Robert-Bosch-Krankenhaus, Stuttgart
}

\title{
Technikbasierte Sturzerkennung und Sturzprädiktion
}

In den vergangenen 2 Jahren wurden in der Sturzpräventionsforschung große Fortschritte erzielt. Hierzu gehören eine neue Metaanalyse der Risikofaktoren von Stürzen [1], Daten zur Epidemiologie von Stürzen in Heimen [2], zur zukünftigen Entwicklung von sturzbedingten Frakturen in Deutschland [3] sowie zur gesundheitsökonomischen Bewertung der Sturzprävention $[4,5]$. Im Frühjahr 2012 wurde erstmals ein Health-Technology-Assessment (HTA)-Bericht zum Thema Sturzprävention veröffentlicht, der - wenn auch kontrovers diskutiert - Aufmerksamkeit auf das Thema gelenkt hat. Auch in den nächsten Monaten sind sehr wichtige Publikationen angekündigt: Die beiden relevanten Cochrane-Reviews zum Thema Sturzprävention werden als Update erscheinen und das Robert-Koch-Institut hat erstmals repräsentative Sturzdaten für den ambulanten Raum erhoben.

Die Europäische Kommission hat Sturzprävention zu einem Hauptziel der Prävention erklärt (CIP-ICT_PSP-2012-6). Hierzu wird ab dem Jahr 2013 das Prevention of Falls Network for Dissemination (ProFouND) gefördert. Die politische Diskussion in Deutschland hat dies ebenfalls erkannt (http://www.gesundheitsziele.de// $\mathrm{cms} /$ medium/814/Gesund_aelter_werden_020512.pdf). Die Themen Bewegungsförderung und Sturzprävention Älterer sind dabei explizit als prioritäre Ziele benannt (S. 44 Abs. 1). Das Aktionsbündnis Patientensicherheit hat das Sturzgeschehen im Krankenhaus als derzeitigen Arbeitsschwerpunkt festgelegt (http://www.aktionsbuendnis-patientensicherheit.de). Der Experten- standard Sturzprophylaxe in der Pflege soll Ende 2012 überarbeitet vorgestellt werden. Im März 2012 wurde erstmals eine nationale Tagung zum Thema Sturzprävention durchgeführt (http://www.sturzpraevention2012. de). Die Diskussion mit den Leistungsträgern wird Ende des Jahres fortgesetzt.

Diese Erfolgsbilanz darf aber nicht verkennen, dass es noch viele Wissenslücken gibt und erhebliche Verbesserungspotenziale vorhanden sind. Hierbei können technische Ansätze einerseits helfen, unser Verstehen des Sturzgeschehens (Biomechanik) und der Risikofaktoren (Prädiktion) zu verbessern [6]. Wenn dies gelingt, ist auch davon auszugehen, dass sich neue Interventionsansätze entwickeln bzw. bestehende Ansätze verbessern lassen. Andererseits können über eine bessere Sturzerkennung unter Verwendung von raumbezogenen oder mobilen Systemen die Rettungszeiten der Betroffenen verkürzt werden. Die neuen technischen Entwicklungen im Bereich der Informations- und Kommunikationstechnologien und der Mikrosystemtechnik ermöglichen begleitende Messungen erst seit Kurzem ausreichend präzise und lang genug.

Ein zentraler Aspekt der Sturzprävention sollte es sein, dass die Maßnahmen die Teilhabechancen erhöhen und körperliche Aktivität verbessern. Dies wurde bereits 2005 vom Prevention of Falls Network Europe gefordert [7]. Mit dem Einsatz der objektiven Aktivitätserfassung lässt sich diese Frage beantworten. Ein Beispiel, dass die Bedeutung einer differenzierten Bewertung eindrucksvoll unterstreicht, ist die Heidelberger Demenz-Trainingsstudie [8]. Hierbei konnte gezeigt werden, dass das Programm die körperliche Aktivität erhöht hat, die Zahl der Stürze gemessen über die Zeit (12 Monate) sich aber nicht zwischen der Interventions- und Kontrollgruppe unterschied. Die meisten derzeitigen Metaanalysen, HTA-Berichte oder systematische Reviews würden dieses Programm daher als negativ oder wenig aufschlussreich bezeichnen. Dies muss aber aus klinischer Sicht und Patientenperspektive hinterfragt werden. Wenn die Zahl der Stürze pro gelaufene Meter niedriger ist, kann eine Bewertung auch anders ausfallen. Hier sind technische Ansätze obligatorisch, um diese drängenden Fragen zu beantworten.

Bei der Planung des Schwerpunkts für dieses Heft war es unser Anliegen, aktuelle Studienbeispiele auszuwählen und Modellierungen für das Thema zu präsentieren, die von klinischer Relevanz sind. Hierbei wollten wir bewusst auch „work in progress" präsentieren, um die Leser über aktuelle Entwicklungen zu informieren.

Gietzelt et al. stellen erste Ergebnisse aus dem niedersächsischen Forschungsverbund „Gestaltung altersgerechter Lebenswelten“ vor. Hier wird ein neu konstruiertes sensorbasiertes System zur Sturzerkennung vorgestellt, das einen tragbaren Beschleunigungssensor mit einem optischen Sturzerkennungssystem kombiniert und deren Daten fusioniert, um die Rate der falsch-positiven Alarme zu reduzieren. Die vorgestellte Feldstudie zeigt, dass sich gute Laborergebnisse in 
der Realität nicht unmittelbar replizieren lassen und dass die Anzahl der realen Sturzereignisse - trotz Gefährdung so gering ist, dass die Beobachtungsdauer von hier immerhin insgesamt 108 Tagen noch deutlich zu niedrig für eine abschließende Bewertung des Systems erscheint.

Mellone et al. berichten über einen Smartphone-Ansatz, der das Potenzial hätte, die Durchführung des geriatrischen Assessments zu verändern. Hierbei werden die klassischen Testverfahren wie der Timed Up \& Go Test mit dem Smartphone instrumentiert. Die Daten werden objektiv gemessen und dann an eine beliebige IP-Adresse übermittelt. In den Zeiten elektronischer Krankenakten hätte dies unschätzbare Vorteile. Darüber hinaus haben Smartphone-Ansätze das Potenzial, über lange Zeit auch außerhalb des Hauses Daten zu ermitteln und zu übertragen. Damit sind sie zumindest theoretisch geeignet, für die Sturzerkennung und den Alarm eine Schlüsselfunktion zu übernehmen. Selbstverständlich wird dies noch viel Arbeit erfordern, um die Validität und die Praktikabilität des Ansatzes zu testen. Ein entscheidender Vorteil könnte hierbei die fehlende Stigmatisierung sein.

Die Arbeit von Shany et al. beschäftigt sich mit dem Einsatz eines sensorbasierten Sturzrisikoassessments. Der Ausgangspunkt hierbei ist zum einen die Überlegung, dass in vielen Settings nicht genügend Zeit vorhanden ist, um eine differenzierte Bewertung der Sturzrisikofaktoren vorzunehmen. Andererseits berichten die Autoren, dass trotz zahlreicher Studien eine gewisse Frustration eingetreten ist, dass Sturzrisikoskalen häufig nicht besser als die Globalbewertung eines erfahrenen Untersuchers abschneiden und daher vielerorts ein gewisser diagnostischer Nihilismus eingekehrt ist. Das sensorbasierte Sturzrisikoassessment hat auch hier ein Potenzial, da eine zeitnahe Erfassung des Bewegungsverhaltens möglich ist. Tinetti bezeichnet dies als die Analyse der präzipitierenden Risikofaktoren, wohingegen das klassische Assessment nur die prädisponierenden Risikofaktoren erfasst. Der Ansatz erscheint pragmatisch und innovativ, auch wenn vermutlich Modifikationen nötig sein werden.

Die Darstellung eines modifizierten Sturzmodells (Becker et al.), dass in dem EU-Projekt FARSEEING entwickelt wur- de, hat verschiedene Gründe. Nach mehr als 15 Jahren Arbeit auf diesem Gebiet wird deutlich, dass wir eine zunehmend differenzierte Betrachtung des Themas Sturz brauchen, um in vielen kleinen Schritten weiterzukommen. Robinovitch et al. [9] konnten mit Videodaten zeigen, dass viele unserer Annahmen zu Stürzen von Heimbewohnern oberflächlich und teilweise falsch sind. Sie nennen diese Aufnahmen „video footage“. Die Videoaufzeichnungen zeigen das komplexe Zusammenspiel von intrinsischen und extrinsischen Faktoren bei der Entstehung von Stürzen. Zumindest aus wissenschaftlicher Sicht erscheint es sinnvoll, die einzelnen Phasen eines Sturzes separat zu analysieren und für jede Phase $\mathrm{zu}$ reflektieren, welche Interventionsmöglichkeiten existieren. Auch hierbei ist klar, dass ein Modell validiert werden muss. Allein die Diskussion des Artikels mit mehr als 30 Experten hat gezeigt, dass ein erhebliches Potenzial vorhanden ist, unser Wissen zu erweitern. Den Beteiligten ist aber auch bewusst, dass sich ein Teil dieses Wissens nur mit technischer Unterstützung erfassen lässt. Selbstverständlich sind die Angaben und Geschichten der Patienten genauso bedeutsam, Videodaten werden uns nicht alle Antworten geben können.

Mit diesem ersten Schwerpunktheft "Gerontechnologie“ wollen wir eine Diskussion anregen. Eine Fortsetzung ist einmal im Jahr geplant. Wir freuen uns auf eine Diskussion und Ihre Beiträge.

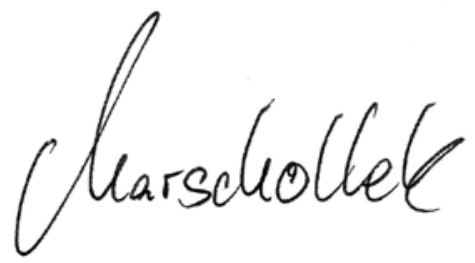

M. Marschollek

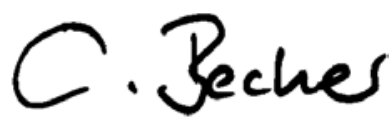

C. Becker

\section{Korrespondenzadresse}

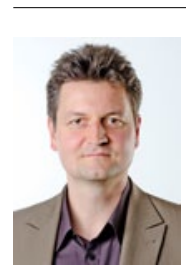

Prof. Dr. med. Dr.-Ing.

M. Marschollek

Peter-L.-Reichertz-Institut für Medizinische Informatik der Technischen Universität

Braunschweig und der

Medizinischen Hochschule Hannover

Carl-Neuberg-Str. 1

30625 Hannover

michael.marschollek@plri.de

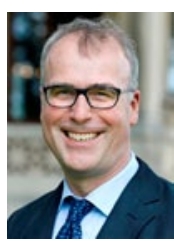

PD Dr. C. Becker

Klinik für Geriatrische

Rehabilitation

Robert-Bosch-Krankenhaus

Auerbachstr. 110,

70376 Stuttgart

Clemens.Becker@rbk.de

\section{Literatur}

1. Deandrea S, Lucenteforte E, Bravi F, et al (2010) Risk factors for falls in community-dwelling older people: a systematic review and meta-analysis. Epidemiology 21:658-668

2. Rapp K, Becker C, Cameron ID, Konig HH, Buchele $G$ (2012) Epidemiology of falls in residential aged care: analysis of more than 70,000 falls from residents of Bavarian nursing homes. J Am Med Dir Assoc 13:187-196

3. Bleibler F, Konnopka A, Benzinger P, Rapp K, Konig $\mathrm{HH}$ (2012) The health burden and costs of incident fractures attributable to osteoporosis from 2010 to 2050 in Germany-a demographic simulation model. Osteoporos Int (Epub ahead of print)

4. Heinrich S, Rapp K, Rissmann U, Becker C, Konig HH (2011) Service use and costs of incident femoral fractures in nursing home residents in Germany: the Bavarian Fall and Fracture Prevention Project (BF2P2). J Am Med Dir Assoc 12:459-466

5. Heinrich S, Weigelt I, Rapp K et al (2012) Fall and fracture prevention based on the National Expert Standard. Implementation and costs in a real world setting in nursing homes. Z Gerontol Geriatr 45:128-137

6. Marschollek M, Rehwald A, Wolf KH et al (2011) Sensor-based fall risk assessment - an expert,to go'. Meth Inf Med 50(5):420-426

7. Lamb SE, Jorstad-Stein EC, Hauer K, Becker C (2005) Development of a common outcome data set for fall injury prevention trials: the Prevention of Falls Network Europe consensus. J Am Geriatr Soc 53:1618-1622

8. Hauer K, Schwenk M, Zieschang T et al (2012) Physical training improves motor performance in people with dementia: a randomized controlled trial. J Am Geriatr Soc 60:8-15

9. Robinovitch SN, Feldman F, Yang Y et al (2012) Video capture of the circumstances of falls in older adults residing in long-term care. Lancet (in press) 